\title{
Aplicación de los nanomateriales en catálisis*
}

\author{
Rodolfo Zanella **
}

\begin{abstract}
RESUMEN: En esta contribución se definen los conceptos fundamentales de la catálisis, se describe la importancia de los procesos catalíticos en la vida cotidiana y en muchos de los procesos que se dan en la naturaleza. Posteriormente se describen las implicaciones de las propiedades físicas, químicas y fisicoquímicas de los nanomateriales en los procesos catalíticos heterogéneos, en los que generalmente el catalizador es un sólido nanoestructurado. Se hace un especial énfasis en el efecto del tamaño y la forma de las nanopartículas sobre la eficiencia de los catalizadores. Asimismo, se describen algunas de las aplicaciones más importantes de los nanocatalizadores, como son el abatimiento de la contaminación atmosférica y del agua, la producción y purificación de combustibles, así como aplicaciones industriales y de química fina para producción de sustancias de muy alto valor comercial como los fármacos, las esencias, los pesticidas, entre otros. Además de describir los procesos nanocatalíticos heterogéneos, se dedica un apartado a los procesos fotocatalíticos, en los que además de un sólido nanoestructurado, se requiere la presencia de luz (ultravioleta o visible) para que se lleven cabo. Estos procesos son de gran importancia en la degradación de compuestos orgánicos e inorgánicos contaminantes del agua, en la producción de hidrógeno y otros combustibles alternativos y en producción de productos químicos de alto valor comercial.
\end{abstract}

PALABRAS CLAVE: catálisis heterogénea, nanomateriales, fotocatálisis, aplicaciones de los nanocatalizadores

ABSTRACT: In this contribution the main concepts of catalysis are defined. The importance of catalytic processes in everyday life and in some processes that occur in the nature are described. The implications of the physical, chemical and physicochemical properties of nanomaterials in heterogeneous catalytic processes are explained. The effect of the size and shape of the nanoparticles on the catalytic efficiency is discussed. In addition, some of the most important applications of nanocatalysts are described, for example the abatement of air and water pollution, the production and purification of fuels, as well as industrial and fine chemicals applications for production of substances of very high commercial value as drugs, essences, pesticides and others. One section is devoted to photocatalytic processes. In these reactions, in addition to the nanostructured solid, the presence of light (ultraviolet or visible) is required to carried out the catalytic process. The photocatalytic reactions are of great importance in the degradation of organic and inorganic pollutants in water, in the production of hydrogen and other alternative fuels, and in the synthesis of chemicals of high commercial value.

KEYWORDS: heterogeneous catalysis, nanomaterials, photocatalysis, nanocatalysts applications

\footnotetext{
* Agradecimientos: a los proyectos PAPIIT IN103513 y Conacyt 130407 por el apoyo financiero otorgado.

** Centro de Ciencias Aplicadas y Desarrollo Tecnológico, Universidad Nacional Autónoma de México. Circuito Exterior S/N, Ciudad Universitaria, A. P. 70-186, Delegación Coyoacán, C.P. 04510, México D. F. Mexico, tel. +52 (55) 56228601.

Correspondencia: (rodolfo.zanella@ccadet.unam.mx).
} 


\section{Fundamentos de la catálisis}

A principios del siglo XIX, cuando comenzaba el estudio científico de la química, se observó, para llevarse a cabo un cierto número de reacciones químicas, la necesidad de la existencia de pequeñas cantidades de sustancias que por sí mismas no formaba parte de la reacción. En 1836, el científico sueco J. J. Berzelius trató de incorporar estas observaciones a los conceptos de la química atribuyendo su acción a lo que llamó su "poder catalítico", a esta acción la llamó catálisis por analogía con el análisis dijo, que es "la separación de las partes componentes de un cuerpo por medio de fuerzas químicas ordinarias. El poder catalítico significa que hay sustancias que por su simple presencia son capaces de despertar las afinidades que están dormidas a esa temperatura..." Posteriormente F. W. Ostwald definió un catalizador como "una sustancia que incrementa la velocidad a la cual el sistema se aproxima al equilibrio, sin ser consumido en el proceso". Esta definición resume la esencia del efecto catalítico y ha superado la prueba del tiempo.

El fenómeno de la catálisis ocurre muy frecuentemente. Nuestra vida y nuestra salud, así como la de todos los seres vivos depende de la acción de catalizadores biológicos llamados enzimas, los cuales generalmente consisten de proteínas, éstas algunas veces tienen átomos metálicos conteniendo un grupo prostético como la clorofila o moléculas hemo (Fe Protoporfina IX). Estos biocatalizadores altamente efectivos están en la cumbre del poder catalítico y todos los catalizadores sintéticos tratan de emularlos. Sin embargo, los catalizadores que se utilizan actualmente en procesos químicos y en control ambiental son compuestos de naturaleza inorgánica que pueden clasificarse en metales, óxidos, sulfuros y ácidos sólidos, aunque en la práctica los catalizadores generalmente contienen compuestos de al menos dos de estas categorías. En particular los metales deben utilizarse en forma de partículas muy pequeñas (nanopartículas) para maximizar su área específica, pero dado que son inestables en ese estado, es necesario separarlos uniéndolos a la superficie de una partícula de un óxido, de tal manera que no puedan estar en contacto unos con otros, este tipo de catalizadores son llamados catalizadores metálicos soportados. Es decir, ocupan una fase diferente a la de la fase fluida en la que están los reactantes y por tanto son llamados catalizadores heterogéneos. Sin embargo, hay muchas especies químicas que pueden actuar catalíticamente cuando se disuelven en una fase líquida en la que se encuentran uno o más de los reactantes, estos catalizadores pueden ser un protón, un ion hidroxilo, una sal o un complejo organometálico. Estos catalizadores son llamados catalizadores homogéneos.

Un catalizador puede acelerar dramáticamente una reacción química, o modificar la distribución de productos, favoreciendo la producción de uno específico, sin ser consumido durante la reacción. Los catalizadores funcionan proporcionando un mecanismo (alternativo) que involucra un estado de transición diferente y una menor energía de activación (figura 1) (Bond y 
FIGURA 1. Energía necesaria para llevar a cabo la reacción en reacciones catalizadas y no catalizadas.

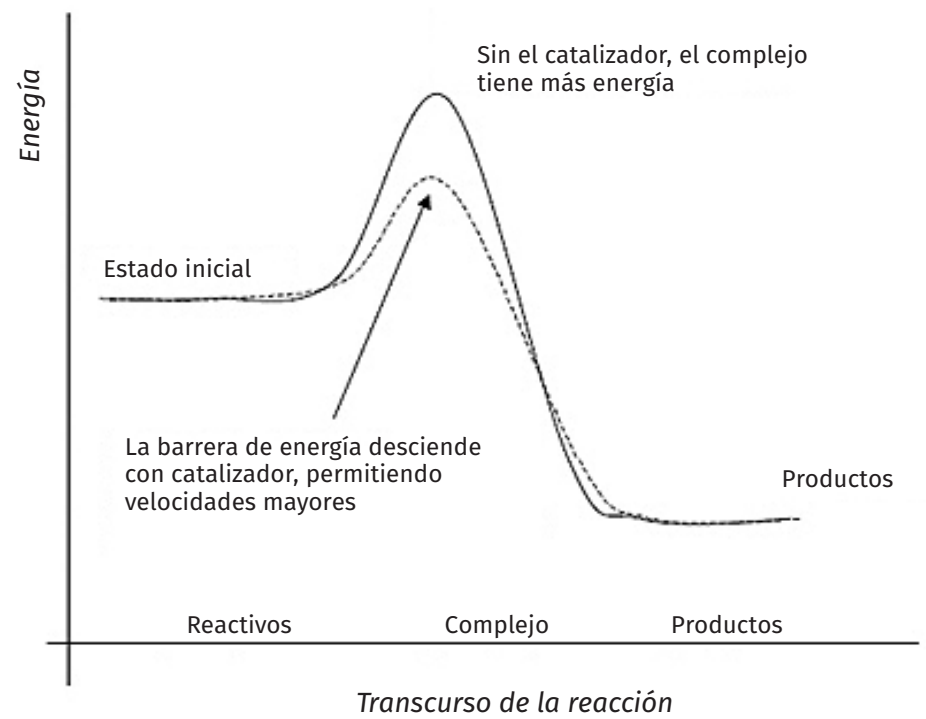

Fuente: Cortesía del Ing. Yamin Arzola Pedroza.

Thompson, 1999; Scott et al., 2003). Por lo tanto, más colisiones moleculares tienen la energía necesaria para alcanzar el estado de transición. En consecuencia, los catalizadores permiten reacciones que de otro modo estarían bloqueadas o desaceleradas por una barrera cinética. El catalizador puede aumentar la velocidad de reacción o la selectividad, o permitir que la reacción ocurra a menores temperaturas.

Una de las características principales de los catalizadores es que la posición de equilibro que se espera para una reacción catalizada es exactamente la misma que la que se esperaría para la reacción en ausencia del catalizador, esto es porque la constante de equilibrio está determinada por la energía libre de Gibbs del proceso y ésta está fijada por los cambios de entalpía y entropía. Además, la velocidad (en una reacción reversible) tanto de la reacción directa como de la inversa se ven afectadas y se compensan mutuamente (Bond y Thompson, 1999; Scott et al., 2003). Así, un catalizador sólo acelera la velocidad con la que se llega a las condiciones de equilibrio pero es incapaz de modificarlas. Otra de las características de un catalizador es que éste sólo puede actuar sobre reacciones que son termodinámicamente factibles bajo una serie de condiciones especificadas. Además, en reacciones complejas que pueden formar varios productos, los catalizadores pueden promover la producción de uno u otro producto, esta característica de los catalizadores se conoce como selectividad. 


\section{La implicación de los nanomateriales en catálisis}

Uno de los objetivos centrales de la nanociencia es construir pequeñas estructuras para el diseño de materiales avanzados y nanodispositivos de alto rendimiento. Las nanopartículas inorgánicas son particularmente atractivas como piezas de construcción para tales propósitos, debido a sus propiedades ópticas, electrónicas, magnéticas y catalíticas únicas (Daniel y Astruc, 2004; Grieve et al., 2000; Lu et al., 2007; Medintz et al., 2005; Pérez-Juste et al., 2005; Shipway et al., 2000), muchas de las cuales pueden ser moduladas simplemente cambiando su tamaño, forma, o la funcionalización de la superficie de la nanopartícula, sin cambiar la composición del material. A la fecha se han realizado avances significativos utilizando estrategias de química húmeda, para sintetizar nanopartículas de alta calidad de una gran variedad de materiales inorgánicos, incluyendo oro, plata, cobre, óxido de hierro y semiconductores como catalizadores o fotocatalizadores (Daniel y Astruc, 2004; Grieve et al., 2000; Lu et al., 2007; Medintz et al., 2005; Pérez-Juste et al., 2005; Sandoval et al., 2011; Sandoval et al., 2013; Shipway et al., 2000). La manipulación de las condiciones de síntesis permite el control racional de la morfología de las partículas y provee los medios para adaptar las propiedades de los materiales durante el proceso de síntesis. Otro aspecto fundamental de la síntesis de nanopartículas es su estabilización, de tal manera que pueda mantenerse su tamaño y su forma en función del tiempo.

Debido a sus propiedades físicas y químicas únicas, las nanopartículas son con frecuencia descritas como átomos artificiales (Alivisatos, 1996; Banin et al., 1999; Collier et al., 1997). Los avances en los procesos de síntesis han permitido el control preciso sobre los parámetros estructurales que gobiernan la formación de las nanopartículas lo que ha permitido adaptar las propiedades de estos átomos artificiales de acuerdo con su uso específico. La síntesis y el ensamblado modular de nanopartículas permiten explotar sus propiedades únicas, lo que puede llevar a nuevas aplicaciones en catálisis, siendo ésta la aplicación química más importante de las nanopartículas metálicas. Los metales de transición, especialmente los metales preciosos, muestran una gran actividad catalítica para muchas reacciones orgánicas e inorgánicas. Estos materiales presentan propiedades tanto en la catálisis homogénea como en la heterogénea (Bond y Thompson, 1999; Heiz y Landman, 2008; Scott et al., 2003).

Como se mencionó arriba, los catalizadores heterogéneos son aquellos que actúan en una fase diferente que los reactivos. La mayoría de los catalizadores heterogéneos son sólidos que actúan sobre sustratos en una mezcla de reacción líquida o gaseosa. El área específica total del sólido tiene un efecto importante en la velocidad de reacción. Cuanto menor sea el tamaño de partícula del catalizador, mayor es el área específica para una masa dada de partículas (Corain et al., 2008). Los catalizadores heterogéneos suelen estar "soportados", es decir, la fase activa se encuentra altamente dispersa en un 
FIGURA 2. Eventos involucrados en el procesos de conversión de reactantes a productos en catálisis heterogénea.

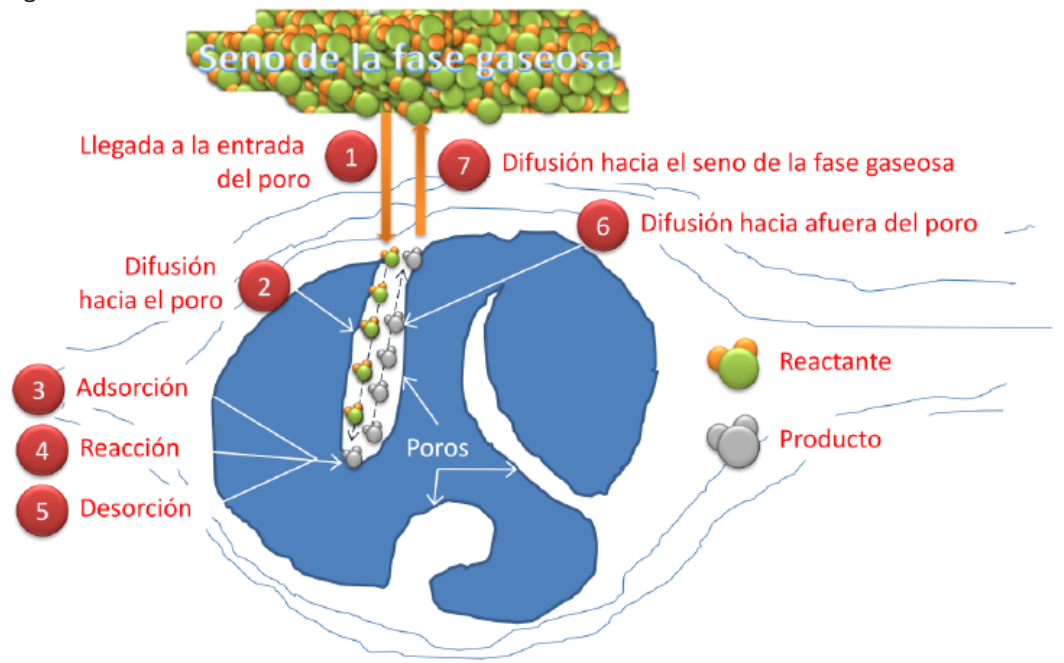

Fuente: Cortesía del M. en C. Alan Soto Sánchez.

material inorgánico, que le proporcione una propiedad adicional al catalizador, por ejemplo, uno de los efectos de los soportes es aportar sitios ácidos o básicos, que puedan interaccionar con el sustrato incrementado la concentración en la superficie del catalizador, además, si la fase activa se encuentra dispersa en un segundo material, muchas veces se mejora la eficacia catalítica, lo cual minimiza su costo. A veces, el soporte es más que una superficie sobre la cual se depositan las fases activas para aumentar su dispersión (Somorjai y Chen, 2001; Somorjai y Li, 2010). Más a menudo, el soporte y la "fase activa" del catalizador interactúan, afectando la reacción catalítica (Bond y Thompson, 1999; Corain et al., 2008; Heiz y Landman, 2008; Scott et al., 2003). En las reacciones heterogéneas están involucradas, además del paso de reacción, otros fenómenos que pueden ser tan o más importantes en el proceso de transformación de reactivos a productos que la reacción en sí. Estos pasos incluyen procesos de difusión de reactivos y productos a través de los poros o los espacios intersticiales del catalizador y procesos de adsorción y desorción superficiales, como se muestra en la figura 2.

\section{Algunas aplicaciones de la catálisis}

La catálisis tiene grandes implicaciones en nuestra vida diaria, pues se estima que el $90 \%$ de todos los productos químicos producidos comercialmente involucran catalizadores en alguna etapa del proceso de su fabricación. En 2012, los procesos catalíticos generaron más de 990,000 millones de dólares en productos a lo largo de todo el mundo. Del mismo modo, la mayoría de los procesos "biológicamente" significativos son catalizados. La investigación 
en catálisis es uno de los principales campos en ciencia aplicada e involucra muchas áreas de la química, especialmente en ciencia de materiales. La catálisis es importante para muchos aspectos de las ciencias del medio ambiente, por ejemplo, en el convertidor catalítico de los automóviles y en la degradación de compuestos orgánicos contaminantes del agua, en el procesamiento de energía, ya que el refinado de petróleo hace un uso intensivo de la catálisis para la alquilación, craqueo catalítico (ruptura de hidrocarburos de cadena larga en trozos más pequeños), reformado de nafta y el reformado con vapor (conversión de hidrocarburos en gas de síntesis). Muchos productos de química fina se preparan a través de procesos catalíticos, los métodos incluyen a los de la industria pesada, así como procesos más especializados que serían prohibitivamente caros a gran escala. Algunos ejemplos son la metátesis de olefinas usando el catalizador de Grubbs, la reacción de Heck, y la reacción de Friedel-Crafts. Asimismo, debido a que la mayoría de los compuestos bioactivos son quirales, muchos productos farmacéuticos son producidos por catálisis enantioselectiva. Por otro lado, una de las aplicaciones más obvias de la catálisis es la hidrogenación (reacción con el hidrógeno gas) de las grasas usando níquel como catalizador para producir la margarina. Muchos otros productos alimenticios se preparan a través de biocatálisis. Hay cientos de ejemplos más de productos que utilizamos en nuestra vida cotidiana que implican procesos catalíticos.

\section{Nanocatálisis}

A pesar de que la nanociencia sólo recientemente se ha materializado como una nueva área interdisciplinaria de la ciencia, el uso de estructuras en la escala nanométrica ha jugado un papel central en la investigación y el desarrollo de catalizadores por décadas. Un catalizador heterogéneo generalmente consiste en nanopartículas de unos cuantos nanómetros dispersadas en un material altamente poroso que muchas veces también tiene dimensiones nanométricas (Somorjai y Chen, 2001; Somorjai y Li, 2010). La disminución del tamaño de partícula provoca que una gran cantidad de átomos estén disponibles, como sitios activos, en la superficie de las partículas, lo que generará catalizadores altamente activos, pues la actividad química es proporcional al número de especies activas accesibles a los reactivos (Bond et al. 2006; Corain et al., 2008). Así, el objetivo principal de la nanocatálisis es controlar las reacciones catalíticas por medio de la optimización del tamaño, dimensionalidad, composición química y morfología de las nanopartículas que constituyen la fase activa del catalizador (Heiz y Landman, 2008; Wachs, 2001). Esto requiere una comprensión fundamental de cómo y por qué las partículas de un catalizador adquieren una forma y tamaño determinados, de cómo permanecen inmovilizadas en la superficie del soporte, de cómo las nanopartículas responden ante los cambios de presión, temperatura, presencia de una atmósfera determinada (reductora, oxidante, etc.) y de cómo 
todos estos factores afectan la actividad catalítica. Así, el término nanocatálisis se refiere a la aplicación de nanomateriales como catalizadores, esta rama ha experimentado un renovado interés en los últimos años en la comunidad científica mundial a pesar de que está demostrado su existencia y aplicación desde hace siglos por los seres humanos, este nuevo interés viene dado por las propiedades que presentan estos materiales como es su alta superficie expuesta, el alto potencial superficial, conductividad eléctrica y su resistencia mecánica (Heiz y Landman, 2008). Varios factores contribuyeron a que esta rama de la catálisis no se desarrollara tan aceleradamente en décadas pasadas, como, por ejemplo, las condiciones de alta energía necesarias para la síntesis de algunos nanomateriales, como en la técnica de evaporación térmica, depósito químico de vapores o implantación iónica, pero, sobre todo, difícilmente se contaba con resultados relacionados con la implicación de diferentes parámetros de la síntesis, sobre la morfología y la distribución de tamaño de partícula, que ahora gracias a los avances logrados en el desarrollo de nuevos dispositivos, como los microscopios electrónicos en sus diferentes modalidades, que proveen imágenes a escala atómica de las partículas del catalizador, o los aceleradores de partículas, que permiten una caracterización directa de los sitios activos y su distribución en el catalizador, se cuenta con datos valiosos para modular y perfeccionar la síntesis de éstos materiales.

En este contexto, algunas propiedades fisicoquímicas únicas de los nanomateriales pudieron haber pasado desapercibidas, hasta hace dos décadas cuando paralelamente al desarrollo de técnicas avanzadas de síntesis de materiales se contó con avances significativos en el desarrollo de técnicas de caracterización físicoquímica, con lo que se logró correlacionar la reactividad de los catalizadores con el tamaño y morfología de las partículas.

La ingeniería de materiales brinda la tecnología necesaria para ejercer el control requerido sobre el tamaño y la morfología de los nanocatalizadores para aprovechar sus propiedades únicas, estableciéndose diversos métodos de síntesis los cuales están directamente relacionados con las peculiaridades de estos materiales (Allcock, 2008; Wachs, 2001). El desarrollo de nuevos métodos de síntesis basados en métodos químicos en fase líquida, como los métodos coloidales, el depósito precipitación o la química organometálica, los cuales se llevan a cabo en condiciones relativamente suaves y con resultados exitosos, pues se logran distribuciónes de tamaño de partícula metálica muy estrechas y un tamaño inferior a 5-10 nm, lo cual ha permitido estudiar la relación del tamaño con las características catalíticas de nanocatalizadores en toda una serie de reacciones heterogéneas (Bond y Thompson, 1999; de-Jong, 2009; Scott et al., 2003). Así, se ha mostrado que del método de síntesis utilizado dependen en gran medida la actividad catalítica, selectividad y desactivación de los nanocatalizadores. Este hecho ha sido reseñado en varios trabajos (Bond y Thompson, 1999; de-Jong, 2009; Scott et al., 2003). 


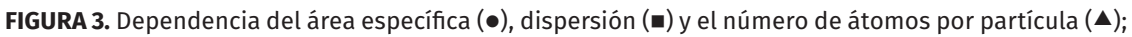
en función del diámetro de partículas esféricas de oro.

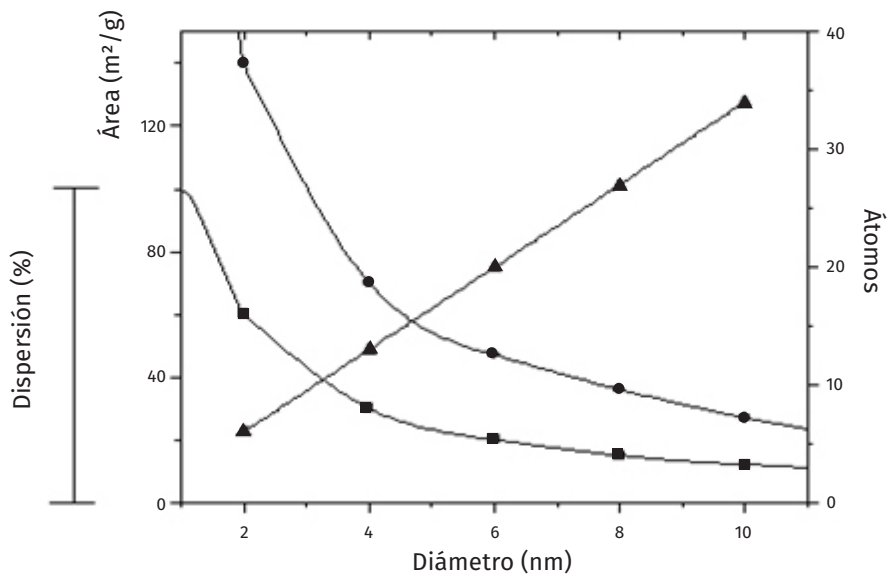

Fuente: Cortesía del Ing. Yamin Arzola Pedroza.

La notable cantidad de átomos disponibles como sitios activos en la superficie de las partículas de tamaño reducido genera catalizadores altamente activos, ya que la actividad química es proporcional al número de especies activas accesibles al sustrato. En la figura 3, se muestra la dependencia del área específica, de la dispersión y del número de átomos por partícula en función del tamaño de partículas esféricas de oro. Otra característica de este tipo de sistema es la interacción soporte-metal que estabiliza las partículas metálicas, por lo cual son menos propensas a sufrir la sinterización y por tanto a desactivarse por este mecanismo. Una de las ramas de la nanocatálisis se orienta a la síntesis de partículas metálicas nanométricas soportadas, como catalizadores novedosos.

Algunos estudios han relacionado no sólo el tamaño, sino también la forma de las nanopartículas con la actividad catalítica, así, por ejemplo, se sabe que las nanopartículas de Au soportadas en óxidos son muy activas en la reacción de oxidación de monóxido de carbono con oxígeno molecular, $\left(\mathrm{CO}+1 / 2 \mathrm{O}_{2} \rightarrow \mathrm{CO}_{2}\right.$ ) (Haruta, 2002; Haruta et al., 1993). A pesar de que el oro es el más noble de todos los metales de transición, cuando se logra obtener en forma de nanopartículas de menos de $5 \mathrm{~nm}$, soportado en la superficie de varios óxidos, por ejemplo $\mathrm{TiO}_{2}$, muestra actividades excepcionalmente altas hacia la reacción de oxidación de $\mathrm{CO}$ a temperaturas por debajo de la temperatura ambiente. El origen de esta sorprendentemente alta reactividad se ha estudiado intensivamente en los últimos años (Bond et al., 2006; Heiz y Landman, 2008). En este sistema catalítico se ha mostrado que la actividad catalítica de los catalizadores depende fuertemente del tamaño y la morfología de las nanopartículas de oro (Akita et al., 2001; Bamwenda et al.,1997; 
FIGURA 4. Mecanismo de reacción de la oxidación del $\mathrm{CO}$ en el catalizador de oro sobre un óxido metálico.

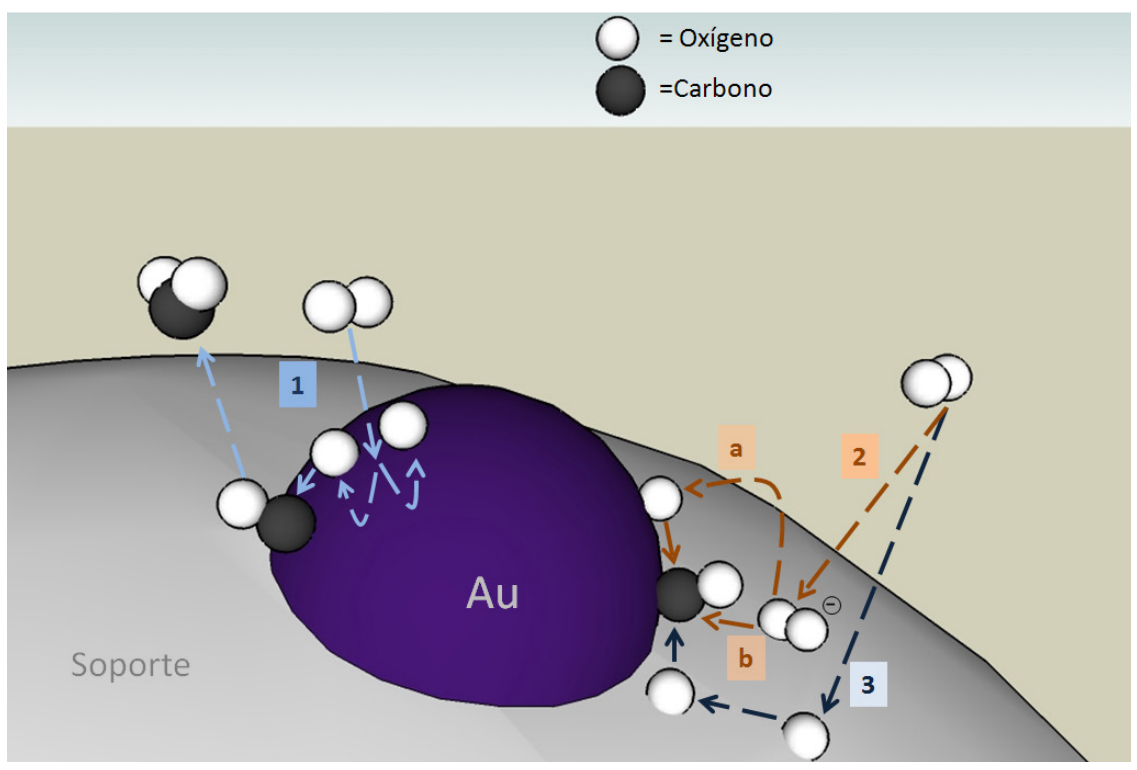

Fuente: Editado por el M. en C. Alan Soto Sánchez de la referencia Schubert et al., 2001.

Okumura et al., 1998; Tsubota et al., 1998; Valden y Goodman, 1998; Valden et al., 1998; Valden et al., 1998; Zanella et al., 2004a; Zanella et al., 2004b). Normalmente, es aceptado que la actividad catalítica del oro aumenta cuando el tamaño de la partícula disminuye, aunque también se ha propuesto que para la oxidación de $\mathrm{CO}$, hay un tamaño óptimo de partícula de alrededor de $3 \mathrm{~nm}$ (Choudhary y Goodman, 2005; Valden y Goodman, 1998; Valden, et al., 1998). Referente a la morfología de las partículas catalíticas, se ha mostrado que la actividad catalítica depende fuertemente de la morfología de las partículas de oro (Choudhary y Goodman, 2005). Se ha reportado que las partículas hemisféricas son más activas que las partículas esféricas, pues las primeras tienen un mayor perímetro interfacial con el soporte que las segundas (Haruta, 2002; Haruta y Daté, 2001; Liu y Vannice, 1997). También se ha propuesto que los nanocatalizadores de Au son altamente activos cuando forman arreglos tridimensionales del tipo racimos conformados por agrupaciones de ocho a veinticuatro átomos de Au (Bond et al., 2006; Heiz y Landman, 2008). Estos estudios tuvieron un alto impacto en la comunidad científica mundial, pues se demostró el denominado efecto carga entre las nanopartículas metálicas y el soporte, el cual consiste en la transferencia de carga del soporte a las nanoestructuras, si éstas se localizan en sitios con vacancias de oxígeno, entonces el soporte podría donar carga a las nanopartículas y hacerlas altamente activas en la reacción de oxidación de monóxido de carbono (figura 4), ya que la reacción transcurre según la teoría de 
orbitales moleculares mediante una transferencia de electrones de los orbitales de más alta energía de las nanopartículas al orbital de anti-enlace de más baja energía del CO, lo que ocasiona la disociación del sustrato y la subsecuente reacción. Por el contrario, si las partículas están soportadas en sitios donde el óxido no presenta defectos estructurales la reacción no ocurre.

\section{Fotocatálisis}

Entre los procesos de oxidación avanzada, la fotocatálisis en presencia de un semiconductor irradiado ha probado ser muy efectiva en el campo de remediación ambiental. La fotocatálisis heterogénea está basada en el hecho de irradiar un óxido semiconductor en forma nanométrica con luz de una energía deseada, para iniciar reacciones químicas, mediante la producción de pares electrón-hueco que finalmente generan radicales hidroxilo en la superficie del catalizador (figura 5).

La principal ventaja de la fotocatálisis heterogénea, comparada con métodos convencionales, es que en muchos casos es posible obtener la mineralización completa de la sustancia tóxica, inclusive en ausencia de reactivos adicionales. El papel del fotocatalizador es el de absorber la energía radiante y transformarla en especies altamente reactivas. Un fotocatalizador ideal debe exhibir las siguientes características: (i) altas velocidades de reacción en la zona de energía de banda prohibida (band gap) (ii) fotoestabilidad, (iii) que no tenga actividad química ni biológica, (iv) disponibilidad y bajo costo.

FIGURA 5. Mecanismo general propuesto para la degradación de contaminantes disueltos en agua por medio de fotocatálisis utilizando semiconductores modificados con nanopartículas de oro.

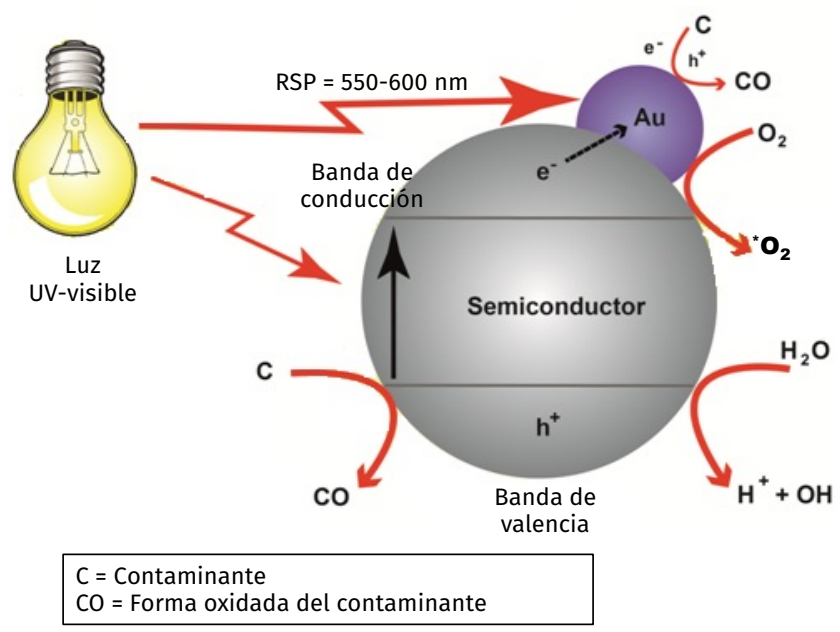

Fuente: Cortesía del Dr. Juan Carlos Durán Álvarez. 
Muchos seminconductores (como $\mathrm{TiO}_{2}, \mathrm{ZnO}, \mathrm{ZrO}_{2}, \mathrm{CdS}, \mathrm{MoS}_{2}, \mathrm{Fe}_{2} \mathrm{O}_{3}, \mathrm{WO}_{3}$, etc.), tanto puros como dopados, han sido examinados y usados como fotocatalizadores para la degradación de contaminantes en aire y agua. $\mathrm{El} \mathrm{TiO}_{2}$ es el semiconductor más utilizado, porque es barato, no es peligroso y su (foto) estabilidad es muy alta.

Algunos tipos de impurezas e imperfecciones pueden afectar drásticamente las propiedades de un semiconductor. De hecho, la conductividad de un semiconductor puede incrementarse significativamente agregando átomos diferentes en la red cristalina (dopado), lo cual genera electrones disponibles en la banda de conducción y huecos en la banda de valencia. Dependiendo del tipo de dopante (donador o receptor) que se agregue al semiconductor los niveles energéticos pueden modificarse y se pueden generar dos tipos de conductores, los tipo " $n$ " cuando el nivel de Fermi se desplaza hacia la banda de conducción, mientras que en los tipo " $p$ " el nivel de Fermi se encuentra más cerca de la banda de valencia. En los semiconductores tipo " $n$ " los electrones pueden pasar más rápidamente a través de ellos, mientras que en los tipo " $p$ " los electrones pueden ser promovidos favoreciendo la formación de huecos.

Las propiedades fotocatalíticas de un semiconductor dependen de la posición de los niveles energéticos, de la movilidad y el tiempo de vida medio de los electrones y huecos fotogenerados, del coeficiente de absorción de luz y de la naturaleza de la interface. Además, la fotoactividad depende de los métodos de preparación del catalizador, que nos permiten variar muchas propiedades fisicoquímicas del semiconductor, controlando la estructura cristalina, el área específica y la distribución de tamaños de partícula (Primo et al., 2011).

En un sistema fotocatalítico, el comportamiento de cada partícula individual del semiconductor es similar al de una celda fotoelectroquímica constituida por un electrodo semiconductor en contacto con un electrodo de un material inerte. En una celda fotoelectroquímica una reacción de oxidación o de reducción puede ocurrir en el electrodo semiconductor, mientras que en una partícula semiconductora inmersa en una solución electrolítica ambas reacciones ocurren simultáneamente por transferencia de huecos de la banda de valencia y de electrones de la banda de conducción. La ventaja de usar suspensiones de polvos semiconductores en forma nanométrica es que las partículas actúan como pequeñas fotoceldas (Tian y Tatsuma, 2005).

El depósito de pequeñas cantidades de metales nobles en forma de nanopartículas en la superficie de las partículas de un semiconductor puede ser usado para incrementar la fotoactividad. En efecto, la velocidad de fotoxidación de los compuestos orgánicos está generalmente limitada por la velocidad de transferencia de electrones del oxígeno adsorbido en la superficie del semiconductor. Entre más electrones estén disponibles para reducir el oxígeno, más altas serán las velocidades de reacción. La figura 6 muestra el efecto de depositar nanopartículas metálicas, principalmente de oro en la 
FIGURA 6. Representación esquemática del mecanismo de la ruptura fotocatalítica de la molécula de agua.

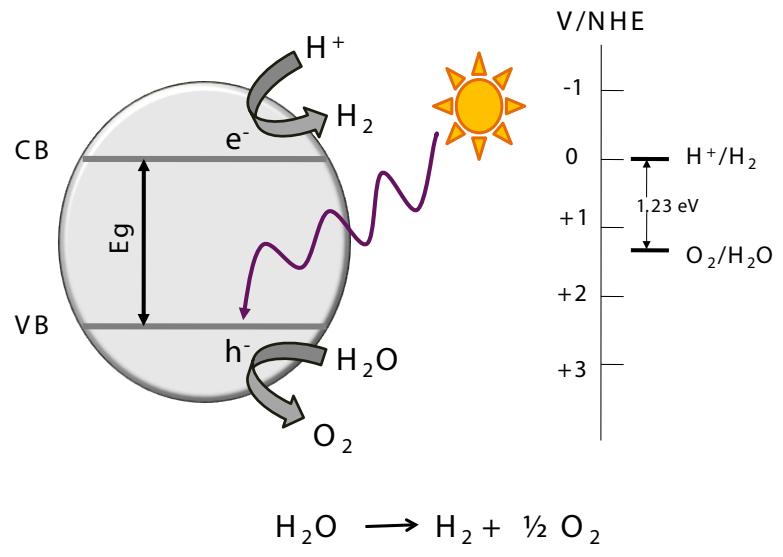

Fuente: Cortesía de la Dra. Socorro Oros Ruiz.

superficie de un semiconductor, estas partículas funcionan como co-catalizadores, que promueven la separación del par electrón-hueco (Hou y Cronin, 2013; Primo et al., 2011).

Es posible explicar el efecto de la presencia de un metal noble comparando una celda fotoelectroquímica y el proceso que ocurre cuando se irradia una partícula de un semiconductor tipo " $n$ " parcialmente cubierto con nanopartículas de un metal. La actividad fotocatalítica de un polvo semiconductor está basada en procesos de oxidación y de reducción que ocurren continuamente en diferentes zonas de la misma partícula. En presencia de metales como $\mathrm{Au}, \mathrm{Ag}, \mathrm{Pd}$ o Pt existen sitios de reacción separados por los pares fotogenerados, de manera similar a una celda consistente de un semiconductor tipo " $n$ " y un contra-electrodo de Pt en el cual ocurre la reducción de las especies oxidadas en el electrodo oxidado mientras que la oxidación de las especies reducidas ocurre en el semiconductor (Hou y Cronin, 2013; Primo et al., 2011).

En las partículas modificadas, los electrones fotogenerados son transferidos sobre las nanoislas metálicas mientras que los huecos permanecen en el semiconductor; esto resulta en la aceleración de los procesos cinéticos, debido a un decremento en la velocidad de recombinación del par electrónhueco. El mismo efecto benéfico puede ser obtenido al cubrir parcialmente la superficie de las partículas del semiconductor con otros óxidos tales como $\mathrm{RuO}_{2}$ o NiO. Otra forma de llevar a cabo separaciones eficientes del par electrón-hueco es usar mezclas de semiconductores, tales como $\mathrm{CdS}$ y $\mathrm{TiO}_{2} \mathrm{o}$ $\mathrm{WO}_{3}$ y WS

El semiconductor más frecuentemente utilizado es sin duda el $\mathrm{TiO}_{2}$ que es producido a gran escala como un pigmento de bajo costo. La actividad fotocatalítica de los 3 polimorfos del $\mathrm{TiO}_{2}$ : anatasa, rutilo y brookita es 
afectada por muchos factores tales como la estructura cristalina, el área específica, la distribución de tamaños de partícula, y la densidad de grupos hidroxilos superficiales. Aunque la posición de las bandas de valencia y de conducción de la anatasa y del rutilo son suficientemente positivas para permitir la oxidación de muchas moléculas orgánicas, la fase anatasa exhibe una más alta actividad, pues el nivel de su banda de conducción es más favorable para la transferencia de electrones, necesaria para la reacción de reducción complementaria. La baja eficiencia del rutilo es principalmente debida a la alta velocidad de recombinación de los pares electrón-hueco y a su baja habilidad para fotoabsorber el oxígeno. Sin embargo, se debe tener en cuenta que el $\mathrm{TiO}_{2}$ más usado como fotocatalizador, Degussa P25 contiene una mezcla de ambos polimorfos (25\% de rutilo y $75 \%$ de anatasa).

Las altas energías de banda prohibida de la anatasa $\left(E_{\mathrm{g}}=3.2 \mathrm{eV}\right)$ y el rutilo $\left(E_{\mathrm{g}}=3.0 \mathrm{eV}\right)$ sólo permite la utilización de radiación con longitud de onda por abajo de aprox. $400 \mathrm{~nm}$, lo que representa aprox. el 5 \% de la luz solar. Un método para incrementar la absorción de luz en la región visible es dopar el $\mathrm{TiO}_{2}$ por la adición de iones de metales de transición. La presencia de estos dopantes induce niveles energéticos en la energía de banda prohibida del $\mathrm{TiO}_{2}$, que permiten la absorción de radiación en la región visible.

La actividad fotocatalítica del $\mathrm{TiO}_{2}$ dopado, generalmente es más baja que la del $\mathrm{TiO}_{2}$ puro, salvo las muestras que contienen W o Mo en concentraciones por debajo del $1 \%$. Este efecto negativo es debido al incremento de la velocidad de combinación de los pares electrón-hueco o al decremento de la longitud del paso de difusión libre medio de los acarreadores de carga. La más alta fotoactividad en presencia de W o Mo puede ser atribuida a la más baja velocidad de recombinación debido a procesos redox que llevan al atrapamiento de electrones fotoproducidos a través de la formación de especies $\mathrm{W}(\mathrm{v})$ y $\mathrm{MO}(\mathrm{v})$, a través de los cuales los electrones son sucesivamente transferidos a las moléculas de oxígeno (Bian et al., 2012; Hou y Cronin, 2013; Primo et al., 2011; Zhou et al., 2012).

Dos de las aplicaciones más importantes en las que se han empleado los procesos fotocatalíticos son la degradación de contaminantes orgánicos y las de producción de hidrógeno a través de la ruptura de la molécula de agua (water splitting) (Oros-Ruiz, et al., 2013a; Oros-Ruiz, et al., 2013b). En el primer caso, la presencia de compuestos orgánicos en aguas residuales, así como su efecto en la salud humana y en la vida acuática, han sido ampliamente reportados. En muchos países, los desechos de industrias, hospitales y efluentes domésticos son descargados directamente a diversos cuerpos de agua sin ningún tratamiento previo, generando contaminación y efectos secundarios. La presencia de analgésicos, antibióticos, antiepilépticos, hormonas y muchos otros productos farmacéuticos han sido reportados como causantes de perturbaciones en la salud humana y en los ecosistemas modificando la reproducción de animales y plantas (Gribson et al., 2007 y 2010; Khan y Ongerth 2004). Por ejemplo, el uso continuo de antibióticos y su 
presencia de bajas concentraciones en el medio ambiente han generado la aparición de genes de resistencia en bacterias hacia este fármaco. Las tecnologías de oxidación avanzada como la fotocatálisis se ha vuelto una alternativa atractiva para la descontaminación de agua; su mecanismo está basado en la absorción de luz para la generación de radicales activos que conducen a la mineralización de materia orgánica. Por otro lado, el depósito de nanopartículas metálicas en semiconductores ha mostrado brindar grandes beneficios a la eficiencia de reacciones fotocatalíticas llevadas a cabo en la degradación de compuestos orgánicos, ya que estas partículas actúan como atractores de electrones, evitando la recombinación del par electrón-hueco y desplazando la absorción del luz hacia longitudes de onda de menor energía (Hou y Cronin, 2013; Primo et al., 2011; Zhou et al., 2012).

Por otro lado, en lo que se refiere a la producción de hidrógeno, como es bien conocido, éste es un combustible alternativo con enorme potencial, pues tiene un muy alto contenido energético por unidad de masa, además de que al «quemarse» sólo genera agua como producto de la combustión. La ruptura de la molécula de agua (water splitting) para producir hidrógeno, por medio de procesos fotocatalíticos ha recibido mucha atención en los últimos años, sobre todo utilizando semiconductores como $\mathrm{TiO}_{2}, \mathrm{ZrO}_{2}, \mathrm{NbTaO}_{3}$, $\mathrm{NaTaO}_{3}$. Particularmente, el $\mathrm{TiO}_{2}$ ha recibido gran atención debido a ser estable, no corrosivo, no tóxico, abundante y económicamente accesible. La capacidad del $\mathrm{TiO}_{2}$ para llevar a cabo reacciones fotocatalíticas puede ser mejorada utilizando nanopartículas metálicas o de óxidos como co-catalizadores (Hou y Cronin, 2013; Oros-Ruiz et al., 2013; Primo et al., 2011; Zhou et al., 2012).

\section{Conclusiones y perspectivas}

Lo descrito hasta aquí muestra que los nanomateriales tienen un enorme potencial como catalizadores o fotocatalizadores de toda una serie de reacciones de abatimiento de la contaminación atmosférica o del agua; de producción y purificación de combustibles como los derivados del petróleo, el hidrógeno y el gas de síntesis, así como en toda una serie de reacciones de generación de productos de alto valor comercial o industrial, como fármacos y productos derivados de la química fina.

Los procesos catalíticos generan utilidades muy importantes a lo largo del mundo y están presentes en muchos de los procesos esenciales para la vida, lo que los convierte en un tema de estudio muy importante y pertinente. La catálisis se apoya cada vez más en la nanotecnología y la ciencia de materiales, para generar catalizadores más eficientes, estables y durables, los campos en los que más se investiga en este momento y en los cuales se prevé se continúe trabajando en el futuro cercano son la producción de biocombustibles, la fotosíntesis artificial, el abatimiento de la contaminación del agua y del aire y la generación de productos de alto valor comercial. Se prevé que en 
el futuro cercano el impacto de los materiales a escala nanométrica sea cada vez mayor en el campo de la catálisis.

\section{Bibliografia}

Akita, T., Lu, P., Ichikawa, S., Tanaka, K. y Haruta, M. (2001) Analytical TEM study on the dispersion of Au nanoparticles in $\mathrm{Au} / \mathrm{TiO}_{2}$ catalyst prepared under various temperatures. Surf. Interf. Anal., 31: 73-78.

Alivisatos, A. P. (1996) Semiconductor clusters, nanocrystals and quantum dots. Science, 271: 933-937.

Allcock, H. R. (2008) Introduction to materials chemistry. Hoboken, New Jersey: Wiley.

Bamwenda, G. R., Tsubota, S., Nakamura, T. y Haruta, M. (1997) The influence of the preparation methods on the catalytic activity of $\mathrm{Pt}$ and gold supported on $\mathrm{TiO}_{2}$ for $\mathrm{CO}$ oxidation. Catal. Lett., 44: 83-97.

Banin, U., Cao, Y. W., Katz, D. y Millo, O. (1999) Identification of atomic-like electronic states in indium arsenide nanocrystal quantum dots. Nature, 400: 542544.

Bian, Z., Tachikawa, T., Kim, W., Choi, W. y Majima, T. (2012) Superior electron transport and photocatalytic abilities of metal-nanoparticle-loaded $\mathrm{TiO}_{2}$ superstructures. J. Phys. Chem. C, 116: 25444-25453.

Bond, G. C., Louis, C. y Thompson, D. T. (2006) Catalysis by Gold (1a. ed., vol. 6). Londres: Imperial College Press.

Bond, G. C., y Thompson, D. T. (1999) Catalysis by gold. Catal. Rev.-Sci. Eng., 41(3 y 4): 319-388.

Collier, C. P., Saykally, R. J., Shiang, J. J., Henrichs, S. E. y Heath, J. P. (1997) Reversible tuning of silver quantum dot monolayers through the metal-insulator transition. Science, 277: 1978-1981.

Corain, B., Schimid, G. y Toshima, N. (2008) Metal nanoclusters in catalysis and materials science. The issue of size control. Amsterdam: Elsevier.

Choudhary, T. V. y Goodman, D. W. (2005) Catalytically active gold: The role of cluster morphology. Appl. Catal. A, 291: 32-36.

Daniel, M.-C. y Astruc, D. (2004) Gold nanopartilces: Assembly, supramolecular chemistry, quantum-size-related properties, and applications toward biology, catalysis and nanotechnology. Chem. Rev. 104: 293-346.

De-Jong, K. P. (2009) Synthesis of solid catalysts. Weinheim: Wiley-VCH.

Gibson, R., Becerril-Bravo, E., Silva-Castro, V. y Jimenez, B. (2007) Determination of acidic pharmaceuticals and potential endocrine disrupting compounds in wastewaters and spring waters by selective elution and analysis by gas chromatography-mass spectrometry. J. Chromatogr. A, (1169): 31-39.

Gibson, R., Durán-Álvarez, J., León Estrada, K. y Jimenez, B. (2010) Accumulation and leaching potential of some pharmaceuticals and potential endocrine disruptors in soils irrigated with wastewater in the Tula Valley. Chemosphere.

Grieve, K., Mulvaney, P. y Grieser, F. (2000) Synthesis and electronic properties of semiconductor nanoparticles/quantum dots. Current Opinion in Colloid and 
Interface Science, 5: 168-172.

Haruta, M. (2002) Catalysis of gold nanoparticles deposited on metal oxides. Cattech, 6: 102-115.

Haruta, M. y Daté, M. (2001) Advances in the catalysis of Au nanaoparticles. Appl. Catal. A, 222: 427-437.

Haruta, M., Tsubota, S., Kobayashi, T., Kageyama, H., Genet, M. J. y Delmon, B. (1993) Low-Temperature oxidation of $\mathrm{CO}$ over gold supp on $\mathrm{TiO}_{2}, \mathrm{a}-\mathrm{Fe}_{2} \mathrm{O}_{3}$ and $\mathrm{Co}_{3} \mathrm{O}_{4}$. J. Catal., 144: 175-192.

Heiz, U. y Landman, U. (2008) Nanocatalysis. Berlín: Springer.

Hou, W. y Cronin, S. B. (2013) A review of surface plasmon resonance-enhanced photocatalysis. Adv. Funct. Mater., 23: 1612-1619.

Khan S. J.; Ongerth J. E. (2004) Modeling of pharmaceutical residues in Australian sewage by quantities of use and fugacity calculations. Chemosphere. 54: 55-367.

Liu, Z. M. y Vannice, M. A. (1997) $\mathrm{CO}$ and $\mathrm{O}_{2}$ adsorption on model $\mathrm{Au}-\mathrm{TiO}_{2}$ systems. Catal. Lett., 43: 51.

Lu, A. H., Salabas, E. L. y Schuth, F. (2007) Magnetic nanoparticles: Synthesis, protection, functionalization, and applications. Angew. Chem. Int. Ed., 46: 1222-1244.

Medintz, I. L., Uyeda, H. T., Goldman, E. R. y Mattoussi, H. (2005) Quantum dot bioconjugates for imaging, labelling and sensing. Nature Mat., 4: 435-446.

Okumura, M., Nakamura, S., Tsubota, S., Nakamura, T. y Haruta, M. (1998) Deposition of gold nanoparticles on silica by CVD of gold acetylacetonate. Preparation of Catalysts VII (Elsevier), 108: 277.

Oros-Ruiz, S., Zanella, R., López, R., Hernández-Gordillo, A. y Gómez, R. (2013a) Photocatalytic hydrogen production by water/methanol decomposition using $\mathrm{Au} / \mathrm{TiO}_{2}$ prepared by deposition-precipitation with urea. J. Hazard. Mater., 263: 2-10.

Oros-Ruiz, S., Zanella, R. y Prado, B. (2013b) Photocatalytic degradation of trimethoprim by metallic nanoparticles supported on $\mathrm{TiO}_{2}-\mathrm{P} 25$. J. Hazard. Mater., 263: 28-35

Pérez-Juste, J., Pastoriza-Santos, I., Liz-Marzán, L. M. y Mulvaney, P. (2005) Gold nanorods: Syntesis, characterization and applications. Coordination Chemistry Reviews, 249: 1870-1901.

Primo, A., Corma, A. y Garcia, H. (2011) Titania supported gold nanoparticles as photocatalyst. Phys. Chem. Chem. Phys., 1: 886-910.

Sandoval, A., Aguilar, A., Louis, C., Traverse, A. y Zanella, R. (2011) Bimetallic Au$\mathrm{Ag} / \mathrm{TiO}_{2}$ Catalyst Prepared by Deposition-Precipitation. High Activity and Stability in CO Oxidation. J. Catal., 281: 40-49.

Sandoval, A., Louis, C., y Zanella, R. (2013) Improved activity and stability in CO oxidation of bimetallic $\mathrm{Au}-\mathrm{Cu} / \mathrm{TiO}_{2}$ catalysts prepared by deposition-precipitation with urea. Appl. Catal. A, 140-141: 363-377.

Schubert M.M., Hackenberg S., Veen A. C. V., Muhler M., Plzak V., Behm R. J. (2001) CO Oxidation over Supported gold catalysts -"inert" and "active" support materials and their role for the oxygen supply during reaction. J. Catal. 197: 113-122. 
Scott, S. L., Crudden, C. M. y Jones, C. W. (2003) Nanostructured Catalysts. Nueva York: Kluwer Academic.

Shipway, A. N., Katz, E. y Willner, I. (2000) Nanoparticle arrays on surfaces for electronic, optical, and sensor application. Chem. Phys. Chem., 1: 18-52.

Somorjai, G. A., y Chen, P. (2001) Surface materials: The frontier of solid state chemistry. Solid State Ionics, 141-142: 3-19.

Somorjai, G. A. y Li, Y. (2010) Introduction to surface chemistry and catalysis. Hoboken, New Jersey: Wiley.

Tian, Y. y Tatsuma, T. (2005) Mechanisms and applications of plasmon-induced charge separation at $\mathrm{TiO}_{2}$ films loaded with gold nanoparticles. J. Am. Chem. Soc, 127: 7632-7637.

Tsubota, S., Nakamura, T., Tanaka, K. y Haruta, M. (1998) Effect of calcination temperature on the catalytic activity of Au colloids mechanically mixed with $\mathrm{TiO}_{2}$ powder for CO oxidation. Catal. Lett., 56: 131-135.

Valden, M. y Goodman, W. (1998) Structure-activity correlations for Au nanoclusters supported on $\mathrm{TiO}_{2}$. Israel J. Chem., 38: 285.

Valden, M., Lai, X. y Goodman, D. W. (1998) Onset of catalytic activity of gold clusters on titania with the appearance of nonmetallic properties. Science, 281: 1647-1650.

Valden, M., Pak, S., Lai, X. y Goodman, D. W. (1998) Structural studies of CO oxidation over model $\mathrm{Au} / \mathrm{TiO}_{2}$ catal. Catal. Lett., 56: 7-10.

Wachs, I. E. (2001) Characterization of catalytic materials. Nueva York: Momentum Press.

Zanella, R., Giorgio, S., Shin, C. H., Henry, C. R. y Louis, C. (2004a) Characterization and reactivity in $\mathrm{CO}$ oxidation of gold nanoparticles supported on $\mathrm{TiO}_{2}$ prepared by deposition-precipitation with $\mathrm{NaOH}$ and urea. J. Catal., 222: 357367.

Zanella, R., Louis, C., Giorgio, S. y Touroude, R. (2004b) Crotonaldehyde hydrogenation by gold supported on $\mathrm{TiO}_{2}$ : Structure sensitivity and mechanism. J. Catal., 223: 328-339.

Zhou, X., Liu, G., Yu, J. y Fan, W. (2012) Surface plasmon resonance-mediated photocatalysis by noble metal-based composites under visible light. J. Mater. Chem., 22: 21337-21354. 